\title{
On the Diversity Order of Non-Orthogonal Amplify-and-Forward over Block-Fading Channels
}

\author{
Ioannis Krikidis, Member, IEEE, John S. Thompson, Member, IEEE, \\ and Steve McLaughlin, Senior Member, IEEE
}

\begin{abstract}
In this paper, we deal with the performance of nonorthogonal Amplify-and-Forward protocols over block-fading channels (BFNAF), where the source retransmits the same data during cooperation in order to increase spatial diversity. Despite the additional diversity degree that is offered by the channel, channel inversion amplification schemes are not always able to increase the diversity gain of the system due to the high correlation that can result in the two simultaneous transmissions. It is proven that this diversity loss is related to a poor sourcerelay link that via the relay amplification process affects the third available diversity branch corresponding to the second source transmission. In order to resolve this problem, we integrate a fixed gain amplification factor in the BFNAF scheme which efficiently uses the additional diversity degree of the channel and recovers the diversity loss associated with channel inversion schemes. This new BFNAF scheme offers spatial diversity benefits with high reliability and is an appropriate solution for Amplifyand-Forward scenarios in which the source-relay link is not stronger than the relay-destination link. The diversity analysis is based on some well-defined capacity bounds which follow the diversity order of the true capacity and enable theoretical derivations. The enhancements of the proposed schemes are verified through both theoretical results and computer simulations.
\end{abstract}

Index Terms-Cooperative networks, relay channel, block fading, Amplify-and-Forward, spatial diversity.

\section{INTRODUCTION}

C OOPERATION between nodes appears as one of the most interesting paradigms for the deployment of future wireless systems. It enables single-antenna users to "enjoy" space diversity benefits by sharing their physical resources through a virtual transmit and/or receive antenna array. Since the work of Sendonaris et al. [1], [2] introduced the notion of cooperative diversity, a number of relaying protocols have been proposed in the literature [3]-[11]. These schemes can be grouped into three basic classes: Decode-and-Forward (DF), Amplify-and-Forward (AF) and Compress-and-Forward (CF). In DF schemes, the relay decodes the received source message, re-encodes it, and forwards the resulting signal to the destination. In AF schemes, the relay simply scales and retransmits the analog signal waveform received from the source. In contrast, for $\mathrm{CF}$ schemes the relay does not decode the source message, nor does it simply amplify the received signal, but

Manuscript received November 13, 2008; revised July 29, 2009, November 11, 2009, and March 11, 2010; accepted March 24, 2010. The associate editor coordinating the review of this paper and approving it for publication was H.C. Yang.

The authors are with the Institute for Digital Communications, University of Edinburgh, EH9 3JL, UK (e-mail: \{i.krikidis, john.thompson, steve.mclaughlin\}@ed.ac.uk).

Digital Object Identifier 10.1109/TWC.2010.06.081517 it performs something in between these two extremes; it compresses (source coding) the received signal and it then transmits. In practical ad hoc wireless networks, terminals may not able to decode the signal from a certain user, as the codebook is seldom available and the decoding (or the source coding for CF) complexity is unacceptable in most cases. AF transmission requires a lower implementation complexity at the relay node and is the relaying technique considered for this study.

One of the first AF protocols proposed was the Orthogonal Amplify-and-Forward (OAF) proposed by Laneman et al. [3], where AF cooperation is performed in two dedicated orthogonal channels to obey the half-duplex constraint. In order to recover the bandwidth loss that results from the transmission orthogonality, in [6]-[9] the authors proposed the Non-Orthogonal Amplify-and-Forward (NAF) protocol which allows the source to be active during the relay transmission. The NAF scheme uses the available system degrees of freedom more efficiently and offers higher data rate transmissions in comparison to the OAF case. However, its implementation may require the use of complex distributed space time-codes (DSTC) [10] and therefore the complexity appears to be a bottleneck for practical systems. On the other hand, in [11] the authors proposed a modification of the NAF scheme which is appropriate for channels with a coherence time that is equal to the time slot duration. In that protocol, called Block-Fading NAF (BFNAF), the source transmits the same codeword during the cooperative slot in order to efficiently use the additional diversity degree of the system. However, although the proposed BFNAF scheme has a simple implementation and outperforms the NAF scheme at low spectral efficiencies, results in [11] have shown that BFNAF does not provide an additional diversity gain.

This paper offers an extension of the work reported in [11]. It focuses on the diversity behavior of the BFNAF scheme and studies the diversity trends which have been observed in [11]. Based on some well-defined bounds which follow the same diversity order with the BFNAF protocol, we prove that a BFNAF protocol with a channel inversion (CI) amplification factor does not always provide an additional diversity gain due to the high correlation that exists in the simultaneous transmissions. More specifically, we demonstrate that the diversity loss that is observed in a BFNAF CI scheme is related to a poor source-relay link that via the amplification process degrades the third diversity branch of the second source transmission. In order to overcome this limitation, a BFNAF scheme which 


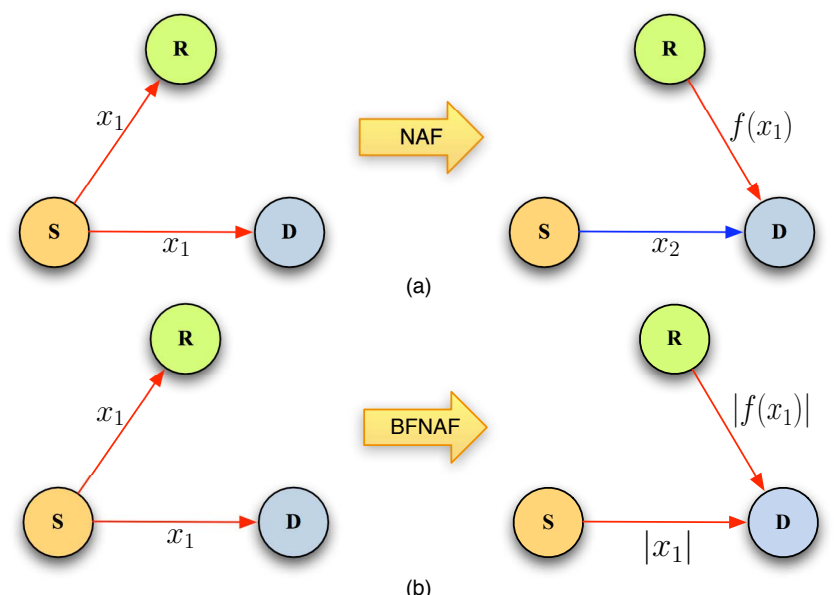

(b)

Fig. 1. The system model for the NAF and the BFNAF protocols; $S$ : source, $D$ : destination, $R$ : relay, $f$ : amplification process.

is based on a constant amplification factor is integrated. The integration of a fixed-gain relay [12]-[14] in the BFNAF protocol, recovers the previously observed loss of diversity gain and efficiently uses all the available diversity degrees of the system. The theoretical analysis of this new protocol is supported by capacity bounds which simplify the diversity analysis. The BFNAF protocol with fixed gain outperforms conventional NAF for the low spectral efficiency regime, uses more efficiently the additional diversity degree of the blockfading channel and enjoys a lower complexity at the relay (independent of channel estimation) and at the destination (only a Maximum Ratio Combiner (MRC) is required).

The paper is organized as follows. Section II introduces the system model and presents the BFNAF protocol. Section III deals with the impact of the amplification factor on the diversity order of the BFNAF scheme. Numerical results are shown and discussed in Section IV, followed by concluding remarks in Section V.

\section{SySTEM MODEL}

Consider a three node cooperative structure consisting of one source $S$, one relay $R$ and one destination $D$. All nodes are half duplex and thus they cannot transmit and receive simultaneously. The physical links between terminals are subject to block-fading, modeled as zero-mean, independent, circularly-symmetric complex Gaussian random variables with a variance $\sigma^{2}$. Consequently, a quasi-static channel which remains constant during the transmission of one data slot but changes independently from one slot to another, is assumed. This system assumption, which corresponds to a coherence time smaller than the frame interval, characterizes some adhoc applications with mobility and is popular in the literature. For the NAF-based protocols, this assumption offers an additional diversity degree which will be efficiently used in our proposed protocols. Furthermore, additive noise is represented as a zero-mean mutually independent, circularly-symmetric, complex Gaussian random sequence with unit variance. A cooperation frame is composed of 2 slots and $x_{i}$ denotes the transmitted source message for the $i$-th slot $(i=1,2)$. Without loss of generality we focus on two sequential time slots which correspond to a basic cooperative frame. The system model can be described as

$$
\begin{aligned}
& y_{D}^{(1)}=\sqrt{P} f_{S, D}^{(1)} x_{1}+n_{S, D}^{(1)}, \\
& y_{R}^{(1)}=\sqrt{P} f_{S, R}^{(1)} x_{1}+n_{S, R}^{(1)}, \\
& y_{D}^{(2)}=\sqrt{P} f_{S, D}^{(2)} x_{2}+\sqrt{P} A f_{R, D}^{(2)} y_{R}^{(1)}+n_{S, D}^{(2)},
\end{aligned}
$$

where $y_{D}^{(t)}, y_{R}^{(t)}$ denote the received symbols at the destination and the relay, respectively, for the $t$-th slot $(t=1,2), A$ denotes the amplification factor (its mathematical definition is given in Section III-A), $f_{k, l}^{(t)}$ is the channel coefficient for the physical link $k \rightarrow l$ at the $t$-th slot, $n_{k, l}^{(t)}$ is the corresponding noise term and $P$ is the transmitted power for each node. Although our study focuses on a symmetric-link configuration (all the links have the same average signalto-noise ratio (SNR)), the proposed techniques can also be applied for the more general non-symmetric case. It is worth noting that the adopted symmetric configuration does not limit the main conclusions of this work and points out all the key issues related to the diversity order of the BFNAF protocol (it is an indicative scenario where the BFNAF CI yields a diversity loss).

The system can be represented by an equivalent channel matrix. Therefore,

$$
\begin{aligned}
{\left[\begin{array}{l}
y_{D}^{(1)} \\
y_{D}^{(2)}
\end{array}\right] } & =\left[\begin{array}{cc}
\sqrt{P} f_{S, D}^{(1)} & 0 \\
\frac{P A f_{S, R}^{(1)} f_{R, D}^{(2)}}{\sqrt{1+P A^{2}\left|f_{R, D}^{(2)}\right|^{2}}} & \frac{\sqrt{P} f_{S, D}^{(2)}}{\sqrt{1+P A^{2}\left|f_{R, D}^{(2)}\right|^{2}}}
\end{array}\right]\left[\begin{array}{l}
x_{1} \\
x_{2}
\end{array}\right]+\mathbf{Z} \\
& \Rightarrow \mathbf{Y}=\mathbf{H X}+\mathbf{Z},
\end{aligned}
$$

where $\mathbf{X}$ is the codeword vector; $\mathbf{H} \in \mathbb{C}^{2 \times 2}$ is the equivalent channel matrix; $\mathbf{Z} \sim C N(0, \mathbf{I})$ is the AWGN at the destination, where I denotes the identity matrix. We note that the scaling factor $\sqrt{1+P A^{2}\left|f_{R, D}^{(2)}\right|^{2}}$ is used at the destination in order to normalize the noise power during the second transmission to 1 .

\section{A. Non-orthogonal Amplify-and-Forward (NAF)}

In contrast to the OAF protocol where the source does not transmit during the cooperative slot, the NAF protocol allows the source to be active during all the frame. In this protocol [7], the codewords $\left(\left[\begin{array}{ll}x_{1} & x_{2}\end{array}\right]\right)$ span two consecutive slot-intervals. In the first phase of the protocol, the source broadcasts the first subcodeword $x_{1}$ and in the second phase, it proceeds with the transmission of the second subcodeword $x_{2}$ (with $x_{2} \neq x_{1}$ ) while the relay retransmits a noisy version of the first subcodeword. In order to decode the transmitted codeword (message), the destination uses the received signals from both phases of the NAF protocol. In this case the corresponding capacity can be written by using the equivalent channel model 
in Eq. (2). Therefore, we have

$$
\begin{aligned}
C_{\mathrm{NAF}}= & \frac{1}{2} \log _{2} \operatorname{det}\left(\mathbf{I}+\mathbf{H H}^{T}\right) \\
= & \frac{1}{2} \log _{2}\left[\left(1+P\left|f_{S, D}^{(1)}\right|^{2}\right)\right. \\
& \times\left(1+\frac{P^{2} A^{2}\left|f_{S, R}^{(1)}\right|^{2}\left|f_{R, D}^{(2)}\right|^{2}+P\left|f_{S, D}^{(2)}\right|^{2}}{1+P A^{2}\left|f_{R, D}^{(2)}\right|^{2}}\right) \\
& \left.-\frac{P^{3} A^{2}\left|f_{S, R}^{(1)}\right|^{2}\left|f_{R, D}^{(2)}\right|^{2}\left|f_{S, D}^{(1)}\right|^{2}}{1+P A^{2}\left|f_{R, D}^{(2)}\right|^{2}}\right] .
\end{aligned}
$$

Fig. 1(a) schematically presents the principles of the NAF protocol. We note that in this work, the NAF scheme is used as a reference protocol and its capacity behavior is based on numerical results.

\section{B. Block Fading NAF (BFNAF)}

The BFNAF scheme is similar to the conventional NAF protocol except that the source retransmits the same packet during the cooperative slot. The principal motivation behind this protocol is the additional diversity branch (link $S \rightarrow D$ of the 2nd slot), which is offered by the considered block-fading channel model. More specifically, the physical link between the source and the destination changes during the cooperative slot $\left(f_{S, D}^{(1)} \neq f_{S, D}^{(2)}\right)$ due to the slot-based block-fading nature of the channel. The source retransmission of the same data via another independent channel increases the receiver diversity and therefore can reduce the error probability. As was shown in [11], this new NAF behavior is interesting for low data rates where the diversity gain is more important than the multiplexing-gain. Using the previous formulation of the conventional NAF scheme, the BFNAF protocol is characterized by the property $x_{2}=x_{1}$. Fig. 1(b) schematically presents the principles of the BFNAF protocol. The behavior of the BFNAF protocol during the cooperative slot can be represented by an equivalent one-dimensional channel which is the algebraic sum of the two individual channels $(S \rightarrow D, R \rightarrow D)$. In this case, the corresponding capacity can be written as

$$
\begin{aligned}
C_{\mathrm{BFNAF}}= & \frac{1}{2} \log _{2}\left(1+\zeta P\left|f_{S, D}^{(1)}\right|^{2}\right. \\
& +\left[\frac{P A f_{R, D}^{(2)} f_{S, R}^{(1)}}{\sqrt{1+P A^{2}\left|f_{R, D}^{(2)}\right|^{2}}}+\frac{\sqrt{P} f_{S, D}^{(2)}}{\sqrt{1+P A^{2}\left|f_{R, D}^{(2)}\right|^{2}}}\right] \\
& \left.\times\left[\frac{P A f_{R, D}^{(2) *} f_{S, R}^{(1) *}}{\sqrt{1+P A^{2}\left|f_{R, D}^{(2)}\right|^{2}}}+\frac{\sqrt{P} f_{S, D}^{(2) *}}{\sqrt{1+P A^{2}\left|f_{R, D}^{(2)}\right|^{2}}}\right]\right)
\end{aligned}
$$

$$
\begin{gathered}
=\frac{1}{2} \log _{2}\left(1+\zeta P\left|f_{S, D}^{(1)}\right|^{2}+\frac{P^{2} A^{2}\left|f_{R, D}^{(2)}\right|^{2}\left|f_{S, R}^{(1)}\right|^{2}}{1+P A^{2}\left|f_{R, D}^{(2)}\right|^{2}}\right. \\
\left.+\frac{P\left|f_{S, D}^{(2)}\right|^{2}}{1+P A^{2}\left|f_{R, D}^{(2)}\right|^{2}}+\frac{2 P \sqrt{P} A \cdot \Re\left\{f_{R, D}^{(2)} f_{S, R}^{(1)} f_{S, D}^{(2) *}\right\}}{1+P A^{2}\left|f_{R, D}^{(2)}\right|^{2}}\right) \\
=\frac{1}{2} \log _{2}\left(\begin{array}{c}
1+\zeta P\left|f_{S, D}^{(1)}\right|^{2}+\left[\frac{P A\left|f_{R, D}^{(2)}\right|\left|f_{S, R}^{(1)}\right|}{\sqrt{1+P A^{2}\left|f_{R, D}^{(2)}\right|^{2}}}\right. \\
\left.+\frac{\sqrt{P}\left|f_{S, D}^{(2)}\right|}{\sqrt{1+P A^{2}\left|f_{R, D}^{(2)}\right|^{2}}}\right]^{2}
\end{array}\right) \text { if } \mathcal{F},
\end{gathered}
$$

where the binary variable $\zeta \in\{0,1\}$ is used to aid the clarity of the presentation, $\Re\{X\}$ denotes the real part of $X \in \mathbb{C}$ and $\mathcal{F} \triangleq \Re\left\{f_{R, D}^{(2)} f_{S, R}^{(1)} f_{S, D}^{(2) *}\right\}=\left|f_{R, D}^{(2)}\right|\left|f_{S, R}^{(1)}\right|\left|f_{S, D}^{(2)}\right|$. In the following analysis the variable $\zeta$ is equal to 1 by default except if defined otherwise. Based on Eq. (4a) we can obtain the equality which is given in Eq. (4b) when the condition $\mathcal{F}$ holds. This capacity expression (Eq. (4b)) can be achieved by using a co-phase alignment between the three links involved $(S \rightarrow D, S \rightarrow R, R \rightarrow D)$ [16], [17] and defines the BFNAF protocol of interest. It is worth noting that in this work, we assume a perfect phase synchronization between the two simultaneous transmissions of the cooperative phase [18], which requires the relay to know perfectly the phase shift between the $S \rightarrow D$ and $S \rightarrow R \rightarrow D$ links. However, a preliminary discussion about the effects of phase ambiguities on the BFNAF performance is presented at the end of this Section.

In order to simplify the analysis of the BFNAF protocol, a useful lower bound is defined as ${ }^{1}$

$$
\begin{gathered}
C_{\mathrm{BFNAF}} \geq \frac{1}{2} \log _{2}\left(1+\zeta P\left|f_{S, D}^{(1)}\right|^{2}+\frac{P^{2} A^{2}\left|f_{R, D}^{(2)}\right|^{2}\left|f_{S, R}^{(1)}\right|^{2}}{1+P A^{2}\left|f_{R, D}^{(2)}\right|^{2}}\right. \\
\left.+\frac{P\left|f_{S, D}^{(2)}\right|^{2}}{1+P A^{2}\left|f_{R, D}^{(2)}\right|^{2}}\right)
\end{gathered}
$$

The above bound includes all the diversity degrees of the protocol (the suppressed term is a correlation of the two diversity branches of the second transmission) and can be used in order to study the diversity behavior of the BFNAF scheme.

1) Phase alignment and complexity issues: The BFNAF protocol requires that simultaneous transmissions phase align so that the signals constructively combine at the destination. This process is called distributed beamforming and emulates a virtual antenna array by transmitting a common message signal towards the direction of the destination [19], [20]. The

\footnotetext{
${ }^{1}$ Based on the identity $(\alpha+\beta)^{2} \geq \alpha^{2}+\beta^{2}$ for $\alpha, \beta \geq 0$ and hence $X+(\alpha+\beta)^{2} \geq X+\alpha^{2}+\beta^{2}$; we note that all the terms in Eq. (4b) are positive.
} 
implementation of distributed beamforming techniques has been widely studied in the literature. In general there are two basic approaches to phase synchronization categorized by the interaction between the sources and the destination. These techniques are (1) closed-loop phase synchronization, where the destination transmits digital feedback to the sources to enable phase alignment and (2) open-loop phase synchronization, where rather than providing feedback, the destination simply transmits an unmodulated sinusoidal beacon and a local interaction between the sources minimizes the interaction with the destination. An overview of different techniques for both categories as well as their related complexity can be found in [19]. However, here, we note the low-complexity technique proposed in [19], [20], where an iterative phase alignment procedure is performed using only a 1-bit feedback transmitted by the destination.

In the case of an imperfect phase alignment at the relay node, a phase offset is introduced in the BFNAF capacity expression. If the parameter $\theta \in[-\pi \pi]$ models the phase ambiguity from the relay co-phasing process, the BFNAF expression given in Eq. (4b) is modified as

$$
\begin{aligned}
& C_{\mathrm{BFNAF}}=\frac{1}{2} \log _{2}\left(1+\zeta P\left|f_{S, D}^{(1)}\right|^{2}+\frac{P^{2} A^{2}\left|f_{R, D}^{(2)}\right|^{2}\left|f_{S, R}^{(1)}\right|^{2}}{1+P A^{2}\left|f_{R, D}^{(2)}\right|^{2}}\right. \\
& +\frac{P\left|f_{S, D}^{(2)}\right|^{2}}{1+P A^{2}\left|f_{R, D}^{(2)}\right|^{2}}+\underbrace{\left.\frac{2 P \sqrt{P} A\left|f_{R, D}^{(2)}\right|\left|f_{S, R}^{(1)}\right|\left|f_{S, D}^{(2)}\right|}{1+P A^{2}\left|f_{R, D}^{(2)}\right|^{2}} \Re\{\exp (j \theta)\}\right)}_{\triangleq \Omega} .
\end{aligned}
$$

The above expression shows that the phase error modifies the last term $(\Omega)$ in the expression for the capacity of BFNAF and could affect the diversity behavior for both CA and CI schemes (i.e. when $\Omega<<0$ ). However, for scenarios where the phase error is limited to $\theta \in[-\pi / 2 \pi / 2]$ (we note that practical systems ensure a small or a moderate phase error), we ensure that $\Omega \geq 0$ and therefore a phase error has no significant effect on the diversity order of the BFNAF CA and BFNAF CI schemes. A further analysis of the phase ambiguity as well as the implementation of the co-phasing process is beyond the scope of this paper and could be considered for future work. However, numerical results in Section IV validate the above intuition and reveal the importance of the phase alignment process.

\section{AMPLIFICATION FACTOR AND DivERSity ANALYSIS}

In this Section, we deal with the effect of the amplification factor on the diversity gain of the BFNAF protocol. We prove that an instantaneous channel-based amplification process limits the performance of the BFNAF scheme and results in a diversity loss. A constant amplification factor seems to overcome this diversity degradation and efficiently uses the available diversity branches. The diversity analysis is based on the behavior of the outage probabilities and the investigation of some well-defined capacity bounds.

\section{A. Amplification factor}

The amplification process is the basic operation which is performed at the relay node. It corresponds to a simple multiplication of the received signal with an amplification factor and a retransmission of the resulting signal to the destination. As this operation is performed in the analogue domain, noise mitigation or other advanced signal processing techniques cannot be applied. In this work, we deal with two different categories of amplification factor; channel inversion (CI) [21] and constant amplification (CA) [12]. The CI consists of an inversion of the channel at the relay and requires the estimation of the instantaneous $S \rightarrow R$ channel. The choice of this gain ensures a constant transmitted power at the relay and therefore the magnitude of the amplification factor decreases with the instantaneous strength of the source-relay channel. However, the CI factor requires a continuous estimate of the channel fading amplitude of the $S \rightarrow R$ link at the relay node which is a critical issue if such channel estimation is not available or if simply the complexity of the relay needs to be kept low [14]. On the other hand, a CA factor does not require an estimate of the instantaneous fading and multiplies the received signal with a fixed gain regardless of the fading amplitude of the $S \rightarrow R$ link. In this work, we adopt a CA factor which is based on the average SNR of the source-relay link. It is worth noting that in practical systems the average channels (which depend on the path loss and shadowing) remain constant for a long period of time and therefore the estimation of the average $S \rightarrow R$ link at the relay node is performed in a sporadic manner by keeping the corresponding complexity low. The considered AF factors can be written as

$$
A=\left\{\begin{array}{cl}
\sqrt{\frac{1}{P\left|f_{S, R}^{(1)}\right|^{2}+1}} & \text { Channel Inversion (CI) } \\
\sqrt{\frac{1}{P \sigma^{2}+1}} & \text { Constant Amplification (CA) }
\end{array}\right.
$$

The amplification factor has a significant impact on the diversity gain of the NAF protocols for the block-fading channel under consideration. Although a CI factor results in a higher SNR and thus a better performance than the CA factor in a conventional OAF environment [12], for the NAF case the behavior of the system needs further investigation. More specifically, in the NAF case, the AF relaying process amplifies the received noise at the relay and degrades the second source transmission which is performed simultaneously with the relaying link. As the second source transmission is not performed in an orthogonal dedicated channel, the amplified noise that results from the AF relaying transmission impacts on the third diversity degree of the system. From a mathematical standpoint, this degradation results in the division of the SNR of the source signal with a noise-related normalization factor in the equivalent channel model and can be observed in equations ( 3 ) and (4b) for the NAF and BFNAF protocols, respectively (please also see [8, Eq. (3)]). In order to analyze the impact of amplification factor on the NAF protocols, we study the outage probability performance for both techniques. We note that the outage probability dominates the overall error probability of the system at high SNRs and its scaling relation with the SNR determines the diversity gain of the system [22], [23]. 


\section{B. Diversity order for BFNAF with $C I$}

By using the capacity expression which is given in Eq. (5), the capacity of the BFNAF protocol with a CI amplification factor is approximated as

$$
\begin{aligned}
& C_{\mathrm{BFNAF}} \approx \frac{1}{2} \log _{2}\left(1+P\left|f_{S, D}^{(1)}\right|^{2}+\frac{P\left|f_{S, R}^{(1)}\right|^{2}\left|f_{R, D}^{(2)}\right|^{2}}{\left|f_{S, R}^{(1)}\right|^{2}+\left|f_{R, D}^{(2)}\right|^{2}}\right. \\
& \left.+\frac{P\left|f_{S, R}^{(1)}\right|^{2}\left|f_{S, D}^{(2)}\right|^{2}}{\left|f_{S, R}^{(1)}\right|^{2}+\left|f_{R, D}^{(2)}\right|^{2}}\right) \\
& =\frac{1}{2} \log _{2}\left(1+P \mu+P \frac{\alpha \beta}{\alpha+\beta}+\frac{P \alpha \gamma}{\alpha+\beta}\right) \\
& \approx \frac{1}{2} \log _{2}\left(1+P \mu+P \min [\alpha, \beta]+\frac{P \alpha \gamma}{\alpha+\beta}\right) \\
& \text { (where } \left.\frac{\alpha \beta}{\alpha+\beta} \approx \min [\alpha, \beta]\right) \\
& \approx \begin{cases}\frac{1}{2} \log _{2}\left(1+P \mu+P \alpha\left[1+\frac{\gamma}{\beta}\right]\right) & \text { if } \alpha<\beta \\
\frac{1}{2} \log _{2}(1+P \mu+P \beta+P \gamma) & \text { if } \beta<\alpha\end{cases}
\end{aligned}
$$

where $\mu \triangleq\left|f_{S, D}^{(1)}\right|^{2}, \alpha \triangleq\left|f_{S, R}^{(1)}\right|^{2}, \beta \triangleq\left|f_{R, D}^{(2)}\right|^{2}, \gamma \triangleq\left|f_{S, D}^{(2)}\right|^{2}$, $\alpha+\beta \approx \max [\alpha, \beta]$ and the approximation given in Eq. (8) is tight at high SNRs and was adopted previously in [24]. In order to analyze the diversity gain of the system, we focus on the outage probability of the above expression for a high SNR regime. More specifically, the outage probability of the BFNAF protocol with a CI amplification factor is given as

$$
\begin{aligned}
& \mathbb{P}\left\{C_{\mathrm{BFNAF}} \leq R_{0}\right\} \approx \underbrace{\mathbb{P}\{\alpha<\beta\}}_{\triangleq \pi_{0}} \underbrace{\mathbb{P}\left\{\mu+\alpha\left[1+\frac{\gamma}{\beta}\right] \leq \frac{\Delta}{P}\right\}}_{\rightarrow\left(\frac{\Delta}{P}\right)^{2}} \\
& +\underbrace{\mathbb{P}\{\beta<\alpha\}}_{\triangleq 1-\pi_{0}} \underbrace{\mathbb{P}\left\{\mu+\beta+\gamma \leq \frac{\Delta}{P}\right\}}_{\rightarrow\left(\frac{\Delta}{P}\right)^{3}} \\
& \approx\left\{\begin{array}{l}
\underbrace{\mathbb{P}\left\{\mu+\beta+\gamma \leq \frac{\Delta}{P}\right\}}_{\rightarrow\left(\frac{\Delta}{P}\right)^{2}} \\
\underbrace{}_{\pi_{0} \underbrace{\mathbb{P}\left\{\mu+\alpha\left[1+\frac{\gamma}{\beta}\right]\right.} \leq \frac{\Delta}{P}\}}
\end{array}\right. \\
& \text { if } \pi_{0}=0 \\
& \text { if } \pi_{0} \neq 0
\end{aligned}
$$

where $\Delta \triangleq 2^{2 R_{0}}-1$ and $R_{0}$ denotes the spectral efficiency.

From the above expression (Eq. (10)) we can see that the outage of the BFNAF CI scheme can be approximated by the sum of two outage probabilities with a weight factor that depends on the quality of the two branches of the relaying link (source-relay and relay-destination links). Although the second term in Eq. (10) follows a diversity order equal to three (sum of three independent exponential random variables), the first term follows a diversity order equal to two as it corresponds to a sum of two independent random variables (Appendix A validates the diversity order of the first term). Therefore the outage probability of the BFNAF CI scheme is a sum of two terms with different diversity orders. The final diversity order of the scheme depends on the weight factor $\pi_{0}$ that defines the relation between these two diversity components. For scenarios that the condition $\pi_{0}=0$ does not hold, the worst term (the first term) in the above expression dominates the error probability at high SNRs $(P \rightarrow \infty)$ [25] and therefore the diversity order of the system becomes equal to two, which corresponds to a diversity loss. For the considered symmetric scenario, we have $\pi_{0}=1 / 2$ and therefore the diversity order of the BFNAF CI scheme is equal to two. This main result shows that, despite the beneficial behavior of the CI approach in the OAF environments, for the BFNAF case a CI approach can result in a high correlation between the two simultaneous diversity branches and therefore a diversity loss. The additional diversity degree which is introduced by the block-fading channel model is not used in an optimal way for scenarios when the source-relay link is not much stronger than the relay-destination link. This conclusion validates the results which have been presented in [11] and requires the investigation of more efficient techniques.

\section{Diversity order for BFNAF with CA}

Based on the previously proposed BFNAF bound in Eq. (5), the capacity of the BFNAF scheme with a CA amplification factor can be written as

$$
\begin{aligned}
& C_{\mathrm{BFNAF}} \approx \frac{1}{2} \log _{2}\left(1+P\left|f_{S, D}^{(1)}\right|^{2}+\frac{P^{2}\left|f_{S, R}^{(1)}\right|^{2}\left|f_{R, D}^{(2)}\right|^{2}}{P+P\left|f_{R, D}^{(2)}\right|^{2}}\right. \\
& \left.+\frac{P^{2}\left|f_{S, D}^{(2)}\right|^{2}}{P+P\left|f_{R, D}^{(2)}\right|^{2}}\right) \\
& =\frac{1}{2} \log _{2}\left(1+P \mu+\frac{P \alpha \beta}{1+\beta}+\frac{P \gamma}{1+\beta}\right) \\
& \approx \begin{cases}\frac{1}{2} \log _{2}(1+P \mu+P \underbrace{\frac{\alpha \beta}{1+\beta}}_{\approx 1}+P \gamma) & \text { if } \beta \leq 1 \\
\frac{1}{2} \log _{2}\left(1+P \mu+P \alpha+P \frac{\gamma}{\beta}\right) & \text { if } \beta>1\end{cases}
\end{aligned}
$$

where the proposed approximation (lower bound) assumes that $1+\beta \approx \max [1, \beta]$. The above expression demonstrates that the capacity of the BFNAF CA scheme is approximated by the sum of three independent random variables for all the values of $\beta$ and thus a diversity gain equal to three is achieved. In order to formulate this main observation, the outage probability of the BFNAF CA protocol can be written as 


$$
\begin{aligned}
\mathbb{P}\left\{C_{B F N A F} \leq R_{0}\right\} & \approx \mathbb{P}\left\{\mu+\frac{\alpha \beta}{1+\beta}+\frac{\gamma}{1+\beta} \leq \frac{\Delta}{P}\right\} \\
& \approx \eta_{0} \underbrace{\mathbb{P}\left\{\mu+\frac{\alpha \beta}{1+\beta}+\gamma \leq \frac{\Delta}{P}\right\}}_{\rightarrow\left(\frac{\Delta}{P}\right)^{3}} \\
& +\left(1-\eta_{0}\right) \underbrace{\mathbb{P}\left\{\mu+\alpha+\frac{\gamma}{\beta} \leq \frac{\Delta}{P}\right\}}_{\rightarrow\left(\frac{\Delta}{P}\right)^{3}} \\
& \rightarrow\left(\frac{\Delta}{P}\right)^{3},
\end{aligned}
$$

where $\eta_{0} \triangleq \mathbb{P}\{\beta \leq 1\}$ (i.e. $\eta_{0}=1-\exp (-1)$ for $\beta \sim \mathcal{C N}(0,1)$ ). Eq. (14) shows that the outage probability of the BFNAF CA corresponds to a (weighted) sum of two outage probabilities with an associated diversity order equal to three; the relation between the two components depends on the parameter $\eta_{0}$. In particular, the case $\beta \leq 1$ is characterized by the sum of two independent exponential random variables $(\mu+\gamma)$ with the term $T \triangleq \frac{\alpha \beta}{1+\beta}$, ([27] proves that the term $T$ corresponds to one diversity branch), and thus provides three independent diversity branches. On the other hand, in Appendix $\mathrm{B}$ we prove that the case $\beta>1$ also corresponds to a sum of three independent diversity branches. As the BFNAF $\mathrm{CA}$ is a sum of two full diversity components, it achieves a diversity order equal to three. Therefore, a CA amplification factor recovers the diversity loss of the CI case that is related to the statistics of the source-relay link and seems to be the appropriate choice for NAF-based protocols in block-fading channels.

\section{Relay saturation}

An important issue of the process of CA amplification is the problem of relay saturation [12]. More specifically, if the first hop of the relaying link has a gain value much higher than its average, the relay amplifier may go into saturation. In this case, an appropriate CA amplification process switches between the CA and CI factor in order to protect the system from potential saturation effects. For the system model under consideration, the switching criterion is the instantaneous normalized SNR of the source-relay link. The CA amplification factor which takes into account the saturation problem follows the definition in Eq. (7) and is written as

$$
A_{\text {sat }}= \begin{cases}\text { CI } & \left|f_{S, R}^{(1)}\right|^{2}>\sigma^{2} \\ \mathrm{CA} & \left|f_{S, R}^{(1)}\right|^{2} \leq \sigma^{2}\end{cases}
$$

The saturation case is a combination of two BFNAF protocols: (a) BFNAF CA which has been proven to use all the diversity degrees of the channel efficiently, (b) BFNAF CI with the constraint $\left|f_{S, R}\right|^{2}>\sigma^{2}$. According to the discussion in Section III.B, the diversity order of the BFNAF CI scheme depends on the relation between two components with a diversity order equal to two and three, respectively. In Appendix $\mathrm{C}$ we demonstrate that, under the considered

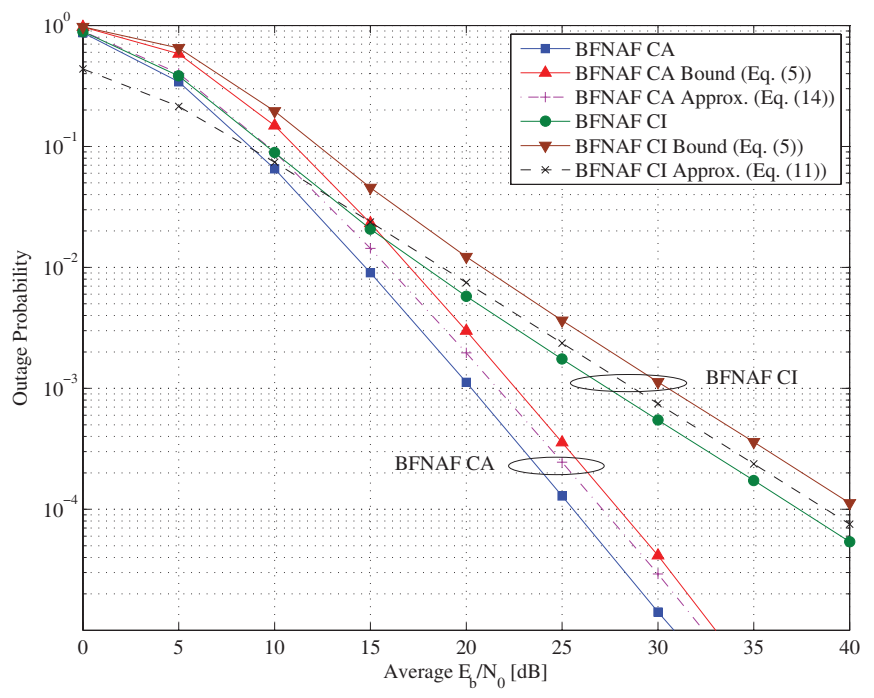

Fig. 2. The outage performance of the BFNAF protocol with $\mathrm{CI}$ and CA amplification factor during the cooperative slot (2nd slot); $\zeta=0, \sigma^{2}=1$, $R_{0}=2 \mathrm{BPCU}, P_{S}^{(2)}=P_{R}$.

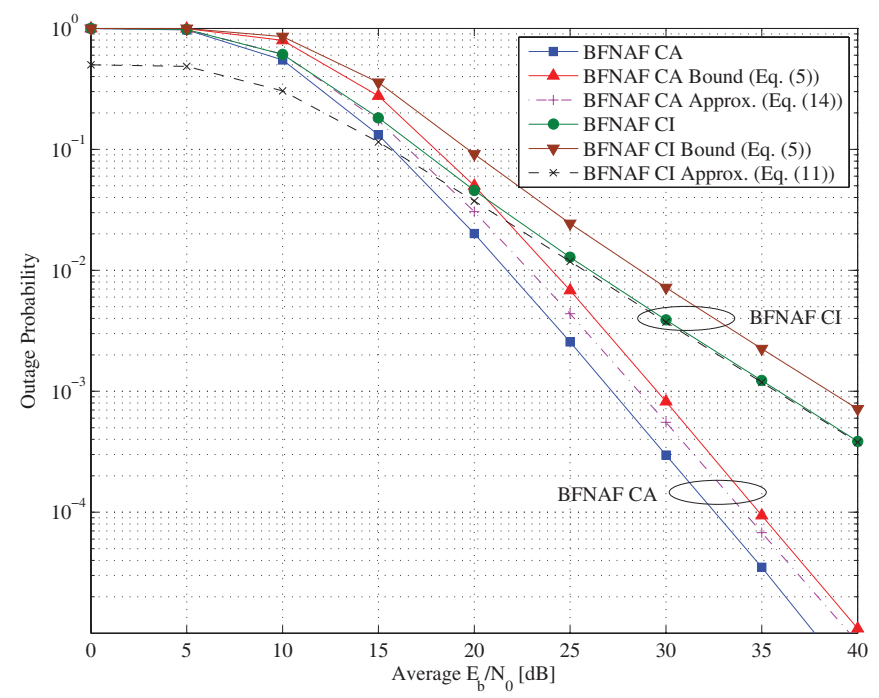

Fig. 3. The outage performance of the BFNAF protocol with CI and CA amplification factor during the cooperative slot (2nd slot); $\zeta=0, \sigma^{2}=1$, $R_{0}=4 \mathrm{BPCU}, P_{S}^{(2)}=P_{R}$.

constraint, the full diversity component dominates the diversity order of a BFNAF CI. As the saturation case is a combination of two protocols with full diversity, it offers a diversity equal to three for all the cases.

\section{NUMERICAL RESUltS}

In this Section, we report numerical results that quantify the performance gains offered by the proposed schemes. The simulation environment focuses on a symmetric configuration with a unit variance $\left(\sigma^{2}=1\right)[15]$ and follows the assumptions of Section III. The performance metric used is the outage probability and the spectral efficiency is expressed in bits per channel use (BPCU).

In order to focus on the simultaneous transmissions of the BFNAF scheme, Figures 2 and 3 deal with the outage probability for the cooperative slot (2nd slot only) and show 
the outage performance for both amplification factors for $R_{0}=2,4 \mathrm{BPCU}$, respectively. The basic idea is to concentrate on the terms which define the overall diversity gain of the system (the first slot corresponds to the first diversity degree) and this setting corresponds to $\zeta=0$ in Eq. (4b). Clearly, a CA amplification factor results in a better outage performance than a CI amplification scheme. A BFNAF protocol with a CI amplification factor yields a diversity gain equal to 1 in contrast to the CA case which provides a diversity gain equal to 2 . This basic observation is in line with the diversity analysis presented above and proves that a CA amplification factor efficiently uses all the diversity degrees of the considered channel. Furthermore, in this figure we plot the simplification steps (approximations) of the presented analysis (equations (11), (14)). As can be seen the proposed approximations follow the same diversity order as the BFNAF scheme, i.e. (are efficient lower bounds), and therefore can be used in order to study their diversity behavior.

Figures 4, 5 and 6 show the outage performance of the complete NAF schemes $(\zeta=1$ in Eq. (4b)) with both amplification factors for three indicative spectral efficiencies equal to $R_{0}=1,2,4 \mathrm{BPCU}$, respectively. In order to have a more reliable comparison a power allocation (PA) policy under a two-slot power constraint is assumed. Therefore, according to [11], an appropriate PA policy for a symmetric configuration is given as $P_{S}^{(1)}=4 / 9 P, P_{S}^{(2)}=1 / 3 P$ and $P_{R}=2 / 9 P$, where $P$ is the total amount of power which is available for the two slots. In [11] we have shown that this PA strategy outperforms conventional symmetric PA and is optimal for both the BFNAF and the NAF protocols in a symmetric configuration (equivalent links). Furthermore, we note that the PA has no impact on the diversity order of the system [7]. The first important observation is that the BFNAF protocol outperforms the NAF scheme at low spectral efficiencies for both amplification factors. In Fig. 4, a gain equal to $0.7 \mathrm{~dB}$ exists for an outage probability equal to $10^{-5}$. This result is in line with the analysis presented in [11] for a CI amplification factor and generalizes this behavior for a CA amplification scheme. However, for high spectral efficiencies the NAF protocol outperforms the BFNAF scheme and its gain increases as the spectral efficiency increases. The interesting observation here is the diversity order of the NAF-based protocols. As can be seen, a CA amplification factor recovers the diversity loss and offers a diversity gain equal to three for NAF and BFNAF protocols. In all the figures the slope of the CA curves show that a CA technique limits the correlation between the two simultaneous transmissions and efficiently uses all the available diversity degrees of the channel. It is worth noting that in all figures, the NAF CA protocol provides a diversity gain equal to three, an observation that is in contrast to conventional NAF studies where the maximum diversity gain is limited to two. However, we note that conventional studies assume that the channel remains constant during cooperation and therefore the available diversity branches are limited to two [7], [8]. Here, we apply the NAF relaying strategy for a conventional block-fading channel, (the source-destination link changes during the second slot), and the illustrated outage probability concerns the full codeword $\left(\left[x_{1} x_{2}\right]\right)$.

Fig. 7.(a) deals with the saturation effects on the diversity

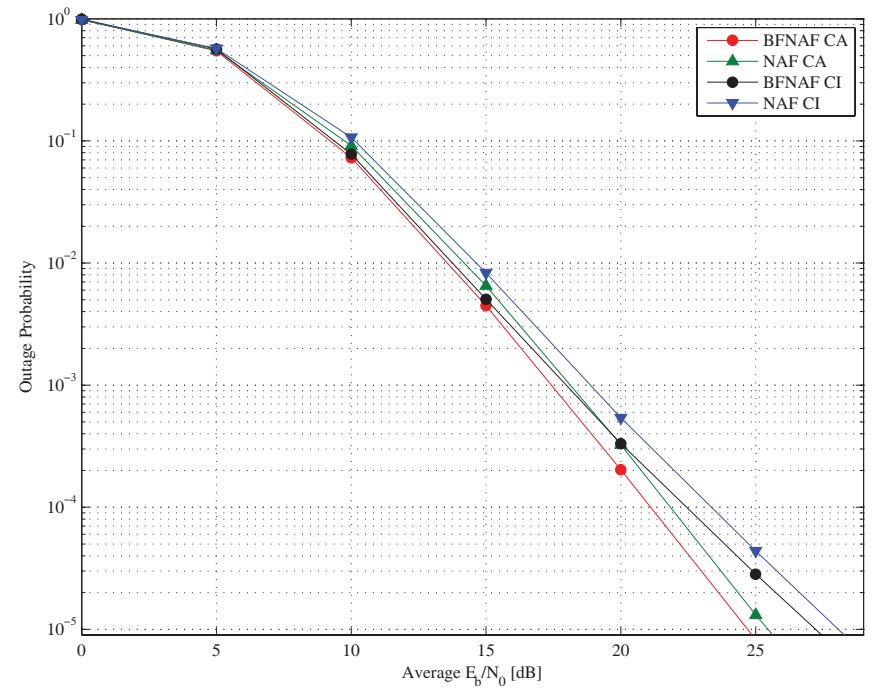

Fig. 4. Outage probability versus SNR for NAF and BFNAF with CI and CA amplification factor (Eq. (4b)); $\zeta=1, \sigma^{2}=1, R_{0}=1 \mathrm{BPCU}$, $P_{S}^{(1)}, P_{S}^{(2)}, P_{R}=\{4 / 9,1 / 3,2 / 9\}$.

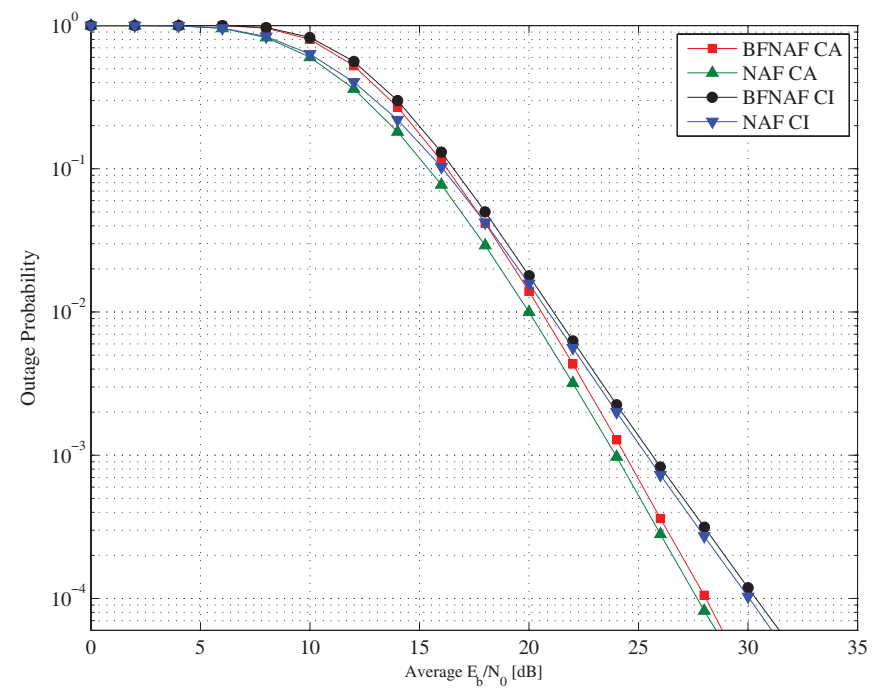

Fig. 5. Outage probability versus SNR for NAF and BFNAF with CI and CA amplification factor (Eq. (4b)); $\zeta=1, \sigma^{2}=1, R_{0}=2 \mathrm{BPCU}$, $P_{S}^{(1)}, P_{S}^{(2)}, P_{R}=\{4 / 9,1 / 3,2 / 9\}$.

order of the CA amplification scheme for the cooperative slot $(\zeta=0$ in Eq. (4b)). As can be seen, switching between the $\mathrm{CI}$ and the CA factors has no impact on the diversity gain of the CA scheme and thus the diversity order continues to equal two. Close inspection of the curves for the BFNAF CA and the saturation case (Fig. 7.(b)) shows that the saturation results in a minimal performance degradation at low SNRs. This behavior is in line with the results presented in [12, Sec. $\mathrm{V}]$ and is based on the fact that the BFNAF CA scheme has a larger amplification gain than the BFNAF CI scheme with $\left|f_{S, R}^{(1)}\right|^{2}>1$ at the low SNR regime (BFNAF CA corresponds to a higher average amplification gain than BFNAF CI with $\left|f_{S, R}^{(1)}\right|^{2}>1$ at low SNRs based on Eq. (7)); however, this gain becomes negligible as the SNR increases. In order to support this observation and validate the related analysis, Fig. 


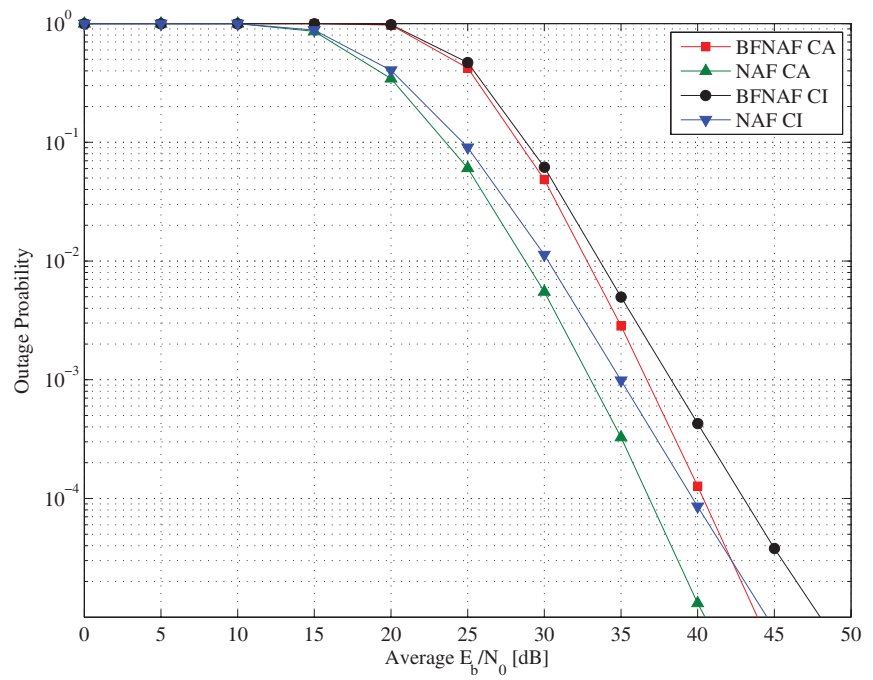

Fig. 6. Outage probability versus SNR for NAF and BFNAF with CI and CA amplification factor (Eq. (4b)); $\zeta=1, \sigma^{2}=1, R_{0}=4 \mathrm{BPCU}$, $P_{S}^{(1)}, P_{S}^{(2)}, P_{R}=\{4 / 9,1 / 3,2 / 9\}$.

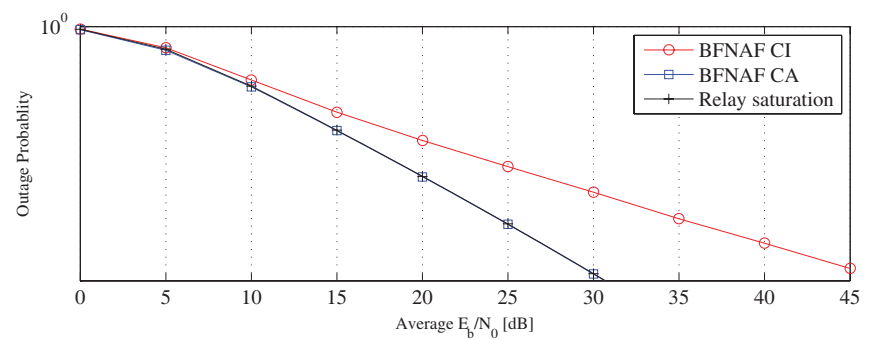

(a)

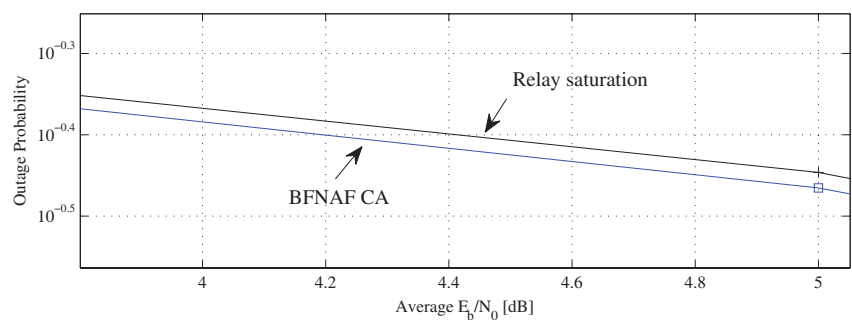

(b)

Fig. 7. Effect of relay saturation on the diversity order of the CA amplification scheme during the cooperative slot (2nd slot); $\zeta=0, \sigma^{2}=1$, $R_{0}=2$ BPCU, $P_{S}^{(2)}=P_{R}$.

8 deals with the diversity order of a BFNAF CI scheme with the constraint $\left|f_{S, R}^{(1)}\right|^{2}>1$. The constraint compensates the diversity loss of the standard BFNAF CI scheme and offers a diversity gain equal to two (for the case that $\zeta=0$ in Eq. (4b)). As the saturation case is switching between two protocols with full diversity gain (BFNAF CA and BFNAF CI with $\left|f_{S, R}^{(1)}\right|^{2}>1$ ), it follows the same diversity order as those protocols.

In Fig. 9 we present the effects of the phase ambiguity on the outage performance of the BFNAF schemes during the cooperative slot (corresponds to $\zeta=0$ in Eq. (6)). More specifically, we compare the outage performance of the BFNAF CI, the BFNAF CA and the saturation case for some indicative phase errors $\left(\theta=0^{\circ}, 60^{\circ}, 90^{\circ}, 120^{\circ}, 180^{\circ}\right)$. This figure shows that an imperfect phase alignment at the relay

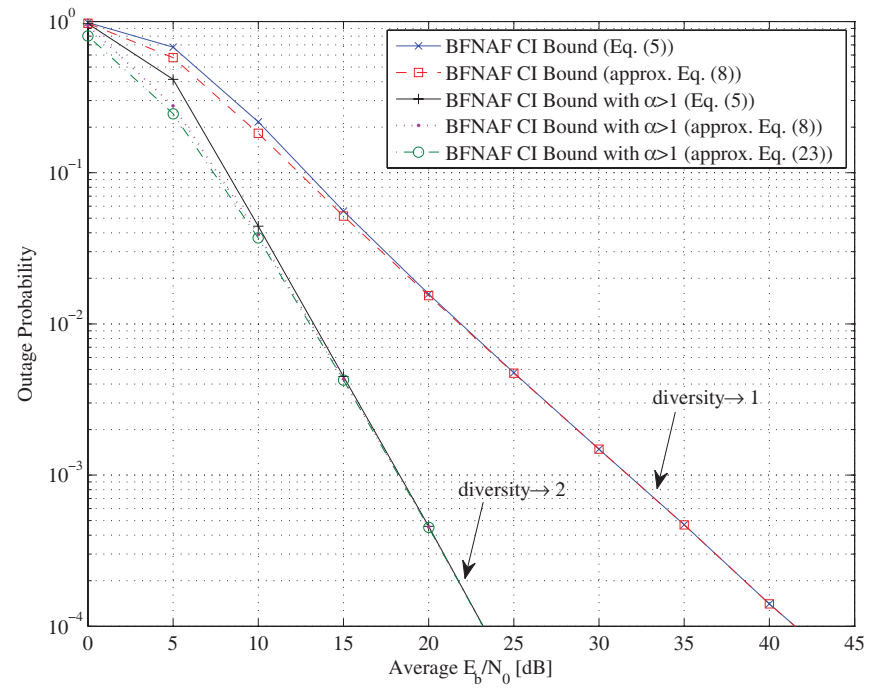

Fig. 8. The diversity order of the BFNAF CI scheme with $\left|f_{S, R}^{(1)}\right|^{2} \equiv \alpha>1$ during the cooperative slot (2nd slot); $\zeta=0, \sigma^{2}=1 R_{0}=2 \mathrm{BPCU}$, $P_{S}^{(2)}=P_{R}$.

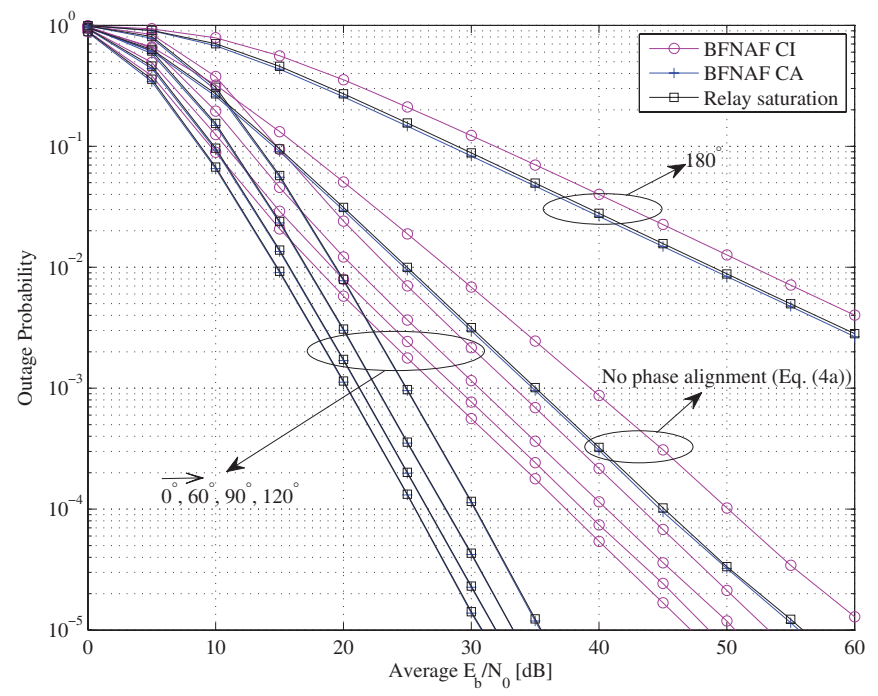

Fig. 9. The impact of the phase offset on the BFNAF schemes; BFNAF CA, BFNAF CI and saturation case (Eq. (4b)); $\zeta=0, \sigma^{2}=1, R_{0}=2$ BPCU, $\theta=0^{\circ}, 60^{\circ}, 90^{\circ}, 120^{\circ}, 180^{\circ}$ and no phase alignment (Eq. (4a)).

node significantly decreases the system performance and can affect the diversity order of the system. In particular, as the phase offset increases, the performance degradation becomes stronger and the outage probability of the system increases. Furthermore, for the scenarios where the phase offset is $|\theta| \leq$ $120^{\circ}$, the diversity order of the system follows our conclusions for perfect phase alignment and thus the diversity order seems to be robust to small and moderate phase ambiguities. It is worth noting that this main observation is in line with [28] where the authors have shown that beamforming gain is robust to phase errors and demonstrated the feasibility of distributed beamforming algorithms. However, for the case that $\theta=180^{\circ}$ (which is a worst case and is used as a reference scenario), the performance of the system significantly decreases and becomes worse than BFNAF without the co-phasing process. 


\section{CONCLUSION}

In this paper, we have analyzed the diversity performance of the BFNAF protocol in block-fading channels. Based on the investigation of some-well defined capacity expressions, it was proven that a channel inversion amplification process at the relay node can degrade the diversity gain of the protocol and result in a diversity loss. This degradation is related to a poor source-relay link that via the amplification process affects the second source diversity branch. In order to recover this loss in performance, a BFNAF protocol which employs a constant amplification factor at the relay was proposed. This scheme protects the simultaneous diversity branches against correlation and efficiently uses all the available diversity degrees of the considered channel. In addition, the saturation case, which consists of switching between a constant amplification factor and a channel inversion according to the quality of the source-relay link, was analyzed. The saturation case achieves full diversity and motivates the investigation of adaptive amplification schemes for the BFNAF transmission.

\section{ACKNOWLEDGEMENTS}

The work reported in this paper has formed part of the Wireless Enabling Techniques work area of the Core 4 Research Programme of the Virtual Centre of Excellence in Mobile and Personal Communications, Mobile VCE, www.mobilevce.com, whose funding support, and that of EPSRC, is gratefully acknowledged. Furthermore, the authors would like to thank the Associate Editor Dr. Hong-Chuan Yang and the anonymous reviewers for their insightful and constructive comments.

\section{APPENDIX A}

\section{THE DIVERSITY ORDER OF THE BFNAF CI WITH $\alpha<\beta$}

In order to simplify the diversity analysis, we focus on the diversity order of the simultaneous transmissions, which corresponds to $\zeta=0$ in Eq. (4b), and a symmetric configuration (without loss of generality).

Let $Y \triangleq \alpha+\frac{\alpha \gamma}{\alpha+\beta}$ a random variable, where $\alpha, \beta, \gamma$ are independent and identically distributed (i.i.d.) exponential random variables with a parameter equal to $\lambda$ with $\alpha<\beta$ (the relay-destination link is much better than the sourcerelay link). We note that $\lambda$ denotes the rate parameter of the exponential distribution and therefore its corresponding mean value is equal to $\sigma^{2}=1 / \lambda$ (the reciprocal of the rate parameter). The cumulative distribution function (CDF) of the random variable $Y$ is given by

$$
\begin{aligned}
P_{Y}(y)= & \mathbb{P}\left\{\alpha+\frac{\alpha \gamma}{\alpha+\beta} \leq y\right\} \\
= & \mathbb{P}\left\{\gamma \leq \frac{(y-\alpha)(\alpha+\beta)}{\alpha}\right\} \quad(\text { with } \alpha \leq y) \\
= & \int_{0}^{y}\left(\int_{\alpha}^{\infty} P_{o}\left(\frac{(y-\alpha)(\alpha+\beta)}{\alpha}\right) p_{o}(\beta) d \beta\right) p_{o}(\alpha) d \alpha \\
& (\text { with } \alpha<\beta) \\
= & \frac{1}{2}-\frac{1}{2} \exp (-2 \lambda y)-\lambda y \frac{1}{2} \exp (-2 \lambda y),
\end{aligned}
$$

where $P_{o}(\cdot), p_{o}(\cdot)$ denote the CDF and the probability density function (PDF), respectively, of the i.i.d. exponential random variables. Therefore the outage probability of the considered BFNAF CI case for high SNRs is

$$
\begin{aligned}
\rho_{\text {out }}= & P_{Y}\left(\frac{\Delta}{P}\right) \\
= & \frac{1}{2}[\underbrace{\left(1-\exp \left(-2 \lambda \frac{\Delta}{P}\right)\right)}_{\approx \frac{2 \lambda \Delta}{P}}-\frac{\Delta}{P} \lambda \exp \left(-2 \lambda \frac{\Delta}{P}\right)] \\
& (\text { with } 1-\exp (-x) \approx x \text { for small } x) \\
\approx & \frac{\lambda \Delta}{2 P}[1+\underbrace{1-\exp \left(-2 \lambda \frac{\Delta}{P}\right)}_{\approx 2 \lambda \frac{\Delta}{P}}] \\
\approx & \frac{1}{2} \frac{\lambda \Delta}{P}+\left(\frac{\lambda \Delta)^{2},}{P}\right]
\end{aligned}
$$

which yields a diversity order equal to

$$
d=-\lim _{P \rightarrow \infty} \frac{\log \rho_{\text {out }}}{\log P}=1 .
$$

Therefore, the BFNAF CI with a strong relay-destination link provides a diversity gain equal to 1 (for the considered $\zeta=0$ case) that corresponds to the diversity loss.

\section{APPENDIX B}

THE DIVERSITy ORDER OF THE BFNAF CA WITH $\beta>1$

As in Appendix A, without loss of generality we focus on a symmetric configuration with $\zeta=0$. Let $\alpha, \beta, \gamma$ be three i.i.d. exponential random variables with parameter $\lambda$ and let the constraint $\beta>1$. The CDF of the random variable $Y \triangleq \alpha+\frac{\gamma}{\beta}$ is given as

$$
\begin{aligned}
& F_{Y}(y)=\mathbb{P}\left\{\alpha+\frac{\gamma}{\beta} \leq y\right\} \\
& =\mathbb{P}\{\gamma \leq y \beta-\alpha \beta\} \quad(\text { with } \alpha \leq y) \\
& =\int_{0}^{y}\left(\int_{1}^{\infty} P_{o}(y \beta-\alpha \beta) p_{o}(\beta) d \beta\right) p_{o}(\alpha) d \alpha \quad(\text { with } \beta>1) \\
& =\exp (-\lambda)[1-\exp (-\lambda y)]-\exp (-\lambda-\lambda y) \ln (1+y) .
\end{aligned}
$$

The outage probability of the BFNAF CA with $\beta>1$ is 
written as

$$
\begin{aligned}
& \rho_{\text {out }}= F_{Y}\left(\frac{\Delta}{P}\right) \\
&= \exp (-\lambda)\left[1-\exp \left(-\lambda \frac{\Delta}{P}\right)\right] \\
& \quad-\lambda \exp \left(-\lambda-\lambda \frac{\Delta}{P}\right) \ln \left(1+\frac{\Delta}{P}\right) \\
& \approx \lambda \exp (-\lambda) \frac{\Delta}{P}+\lambda \exp \left(-\lambda-\lambda \frac{\Delta}{P}\right) \frac{\Delta}{P} \\
&(\text { where } 1-\exp (-x) \approx x, \ln (1+x) \approx x \text { for small x }) \\
& \approx \lambda \exp (-\lambda) \frac{\Delta}{P}\left[1-\exp \left(-\lambda \frac{\Delta}{P}\right)\right] \\
& \approx \lambda^{2} \exp (-\lambda)\left(\frac{\Delta}{P}\right)^{2} \\
& \rightarrow\left(\frac{\Delta}{P}\right)^{2} \cdot
\end{aligned}
$$

Therefore the BFNAF CA scheme with $\beta>1$ achieves full diversity.

\section{APPENDIX C \\ THE DIVERSITY ORDER OF THE BFNAF CI WITH $\alpha>\frac{1}{\lambda_{\alpha}}$}

We focus on the diversity order of the simultaneous transmissions, which corresponds to $\zeta=0$ in Eq. (4b). Let $\alpha, \beta$ two exponential random variables with parameters $\lambda_{\alpha}$ and $\lambda_{\beta}$ (we use different rate parameters for generalization), respectively, and let $\alpha>1 / \lambda_{\alpha}$ where $\lambda_{\alpha}<\frac{P}{\Delta}$ (for large $P$ ). In this case, the outage probability of the BFNAF CI scheme is equal to

$$
\begin{aligned}
\rho_{\text {out }} & =\pi_{0} \underbrace{\mathbb{P}\left\{\alpha+\frac{\alpha \gamma}{\alpha+\beta} \leq \frac{\Delta}{P}\right\}}_{\left.\rightarrow 0 \text { (as } \alpha>\frac{\Delta}{P}\right)}+\left(1-\pi_{0}\right) \underbrace{\mathbb{P}\left\{\beta+\gamma \leq \frac{\Delta}{P}\right\}}_{\rightarrow\left(\frac{\Delta}{P}\right)^{2}} \\
& =\left(1-\pi_{0}\right) \underbrace{\mathbb{P}\left\{\beta+\gamma \leq \frac{\Delta}{P}\right\}}_{\rightarrow\left(\frac{\Delta}{P}\right)^{2}} \\
& \rightarrow\left(\frac{\Delta}{P}\right)^{2},
\end{aligned}
$$

with

$$
\begin{aligned}
\pi_{0} & \triangleq \mathbb{P}\left\{\frac{1}{\lambda_{\alpha}}<\alpha<\beta\right\} \\
& =\int_{\frac{1}{\lambda_{\alpha}}}^{\infty}\left[P_{\alpha}(\beta)-P_{\alpha}\left(\frac{1}{\lambda_{\alpha}}\right)\right] p_{\beta}(\beta) d \beta \\
& =\frac{\lambda_{\alpha}}{\lambda_{\alpha}+\lambda_{\beta}} \exp \left(-1-\frac{\lambda_{\beta}}{\lambda_{\alpha}}\right)
\end{aligned}
$$

where $P_{\alpha}(\cdot)$ is the $\mathrm{CDF}$ of the exponential random variable $\alpha$ and $p_{\beta}(\cdot)$ denotes the PDF of the exponential random variable $\beta$. The probability $\pi_{0}$ corresponds to a strongly decreasing function $g(y) \triangleq \exp (-1) \frac{\exp (-y)}{1+y}$, where $y \triangleq \lambda_{\beta} / \lambda_{\alpha}$ and gives $g(1) \approx 0.0677 \rightarrow 0$ for a symmetric scenario (i.e. symmetric links). The above analysis shows that in contrast to the conventional BFNAF CI case, where the the first term in Eq. (23) dominates the error probability at high SNRs, this term becomes zero in the saturation case. Therefore, the diversity order of the BFNAF CI system depends on the second term that provides full diversity.

\section{REFERENCES}

[1] A. Sendonaris, E. Erkip, and B. Aazhang, "User cooperation diversitypart I: system description," IEEE Trans. Commun., vol. 51, pp. 19271938, Nov. 2003.

[2] A. Sendonaris, E. Erkip, and B. Aazhang, "User cooperation diversitypart II: implementation aspects and performance analysis," IEEE Trans. Commun., vol. 51, pp. 1939-1948, Nov. 2003.

[3] J. N. Laneman, D. N. C. Tse, and G. W. Wornell, "Cooperative divesrity in wireless networks: efficient protocols and outage behavior," IEEE Trans. Inf. Theory, vol. 50, pp. 3062-3080, Dec. 2004.

[4] J. N. Laneman, "Cooperative diversity in wireless networks: algorithms and architectures," Ph.D. dissertation, MIT Publications, Sept. 2002. [Online:] allegro.mit.edu/pubs/posted/doctoral/2002-laneman-phd.pdf

[5] A. Ribeiro, X. Cai, and G. B. Giannakis, "Symbol error probabilities for general cooperative links," IEEE Trans. Wireless Commun., vol. 4, pp. 1264-1273, May 2005.

[6] R. U. Nabar, H. Bölcskei, and F. W. Kneubühler, "Fading relay channels: performance limits and space-time signal design," J. Sel. Areas Commun., vol. 22, pp. 1099-1109, Aug. 2004.

[7] K. Azarian, H. E. Gamal, and P. Schniter, "On the achievable diversitymultilexing tradeoff in half-duplex cooperative channels," IEEE Trans. Inf. Theory, vol. 51, pp. 4152-4172, Dec. 2005.

[8] S. Yang and J. -C. Belfiore, "Optimal space-time codes for MIMO amplify-and-forward cooperative channel," IEEE Trans. Inf. Theory, vo. 53, pp. 647-663, Feb. 2007.

[9] S. Yang and J.-C. Belfiore, "Towards the optimal amplify-and-forward cooperative diversity scheme," IEEE Trans. Inf. Theory, vol. 53, pp. 3114-3126, Sep. 2007.

[10] O. Canpolat, M. Uysal, and M. M. Fareed, "Analysis and design of distributed space-time trellis codes with amplify-and-forward relaying," IEEE Trans. Veh. Technol., vol. 56, pp. 1649-1660, July 2007.

[11] I. Krikidis, J. Thompson, S. McLaughlin, and N. Goertz, "Optimization issues for cooperative amplify-and-forward systems over block-fading channels," IEEE Trans. Veh. Technol., vol. 57, pp. 2868-2884, Sep. 2008.

[12] M. O. Hasna and M.-S. Alouini, "Performance study of dual-hop transmissions with fixed gain relays," IEEE Trans. Wireless Commun., vol. 3, pp. 1963-1968, Nov. 2004.

[13] X. Deng and A. M. Haimovich, "Power allocation for cooperative relaying in wireless networks," IEEE Commun. Lett., vol. 11, pp. 994996, 2005.

[14] D. S. Michalopoulos, A. S. Lioumpas, and G. K. Karagiannidis, "Low complexity amplify-and-forward relaying without channel amplitude estimation," in Proc. IEEE Int. Conf. Commun., Beijing, China, pp. 4295-4299, May 2008.

[15] A. Ribeiro, X. Cai, and G. B. Giannakis, "Symbol error probabilities for general cooperative links," IEEE Trans. Wireless Commun., vol. 4, pp. 1264-1273, May 2005.

[16] B. Zhao and M. C. Valenti, "Practical relay networks: a generalization of hybrid-ARQ," IEEE J. Sel. Areas Commun., vol. 23, pp. 7-18, Jan. 2005.

[17] D. Lee, Y. S. Jung, and J. H. Lee, "Amplify-and-forward cooperative transmission with multiple relays using phase feedback," in IEEE Proc. Vehic. Tech. Conf., pp. 1-5, Sep. 2006.

[18] H. Ochiai, P. Mitran, H. V. Poor, and V. Tarokh, "Collaborative beamforming for distributed wireless ad hoc sensor networks," IEEE Trans. Signal Process., vol. 53, pp. 4110-4124, Nov. 2005.

[19] R. Mudumbai, D. R. Brown, U. Madhow, and H. V. Poor, "Distributed transmit beamforming: challenges and recent progress," IEEE Commun Mag., vol. 47, pp. 102-110, Feb. 2009.

[20] R. Mudumbai, J. Hespanha, U. Madhow, and G. Barriac, "Distributed transmit beamforming using feedback control," IEEE Trans. Inf. Theory, submitted for publication Mar. 2006. [Available online: http://arxiv.org/abs/cs/0603072].

[21] M. O. Hasna and M.-S. Alouini, "End-to-end performance of transmission systems with relays over Rayleigh-fading channels," IEEE Trans. Wireless Commun., vol. 2, pp. 1126-1131, Nov. 2003.

[22] L. Zheng and D. N. Tse, "Diversity and multiplexing: a fundamental tradeoff in multiple-antenna channels," IEEE Trans. Inf. Theory, vol. 49, pp. 1073-1096, May 2003.

[23] D. Tse, Fundamentals of Wireless Communication. Cambridge Univ. Press, 2005 
[24] P. A. Anghel and M. Kaveh, "Exact symbol error probability of a cooperative network in a Rayleigh-fading environment," IEEE Trans. Wireless Commun., vol. 3, pp. 1126-1131, Nov. 2003.

[25] E. Sengul, E. Akkay, and E. Ayanoglu, "Diversity analysis of single and multiple beamforming," IEEE Trans. Commun., vol. 54, June 2006.

[26] A. Bletsas, A. Khisti, D. P. Reed, and A. Lippman, "A simple cooperative divesriy method based on network path selection," IEEE Trans. Wireless Commun., vol. 6, pp. 3450-3460, Sep. 2007.

[27] B. Maham and A. Hjorungnes, "Performance analysis of repetitionbased cooperative networks with partial statistical CSI at relays," IEEE Commun. Lett., vol. 12, pp. 828-830, Nov. 2008.

[28] R. Mudumbai, G. Barriac, and U. Madhow, "On the feasibility of distributed beamforming in wireless networks," IEEE Trans. Wireless Commun., vol. 6, pp. 1-10, Apr. 2007.

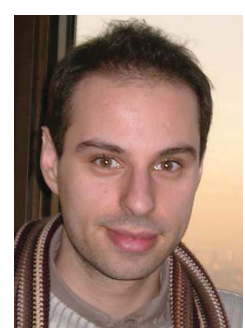

Ioannis Krikidis (S'03-M'07) was born in Athens, Greece, in 1977. He received the diploma in Computer Engineering from the Computer Engineering and Informatics Department (CEID) of the University of Patras, Greece, in 2000, and the M.Sc and Ph.D degrees from Ecole Nationale Supérieure des Télécommunications (ENST), Paris, France, in 2001 and 2005, respectively, all in electrical engineering.

From 2001 to 2002 he served as a Research Associate at the National Capodistrean University of Athens, Athens, Greece and from 2006 to 2007 he worked, as a Post-Doctoral researcher, with ENST, Paris, France. He is currently a Research Fellow in the School of Engineering and Electronics at the University of Edinburgh, Edinburgh, UK. During summer of 2008 and spring of 2009, he was visiting researcher at the University of Notre Dame, IN, USA and the University of Maryland, USA, respectively. His current research interests include information theory, wireless communications, cognitive radio and secrecy communications.

Dr. I. Krikidis is a member of the Technical Chamber of Greece.

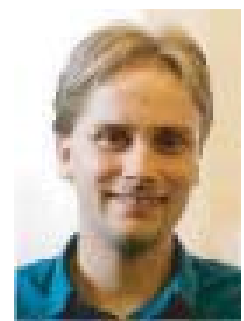

John S. Thompson received the B.Eng. and Ph.D. degrees from the University of Edinburgh, Edinburgh, U.K., in 1992 and 1996, respectively. From July 1995 to August 1999, he was a Postdoctoral Researcher with the University of Edinburgh, which was funded by the U.K. Engineering and Physical Sciences Research Council and Nortel Networks. In September 1999, he was a Lecturer with the School of Engineering and Electronics, University of Edinburgh, where, since October 2005, he has been a Reader. He has authored approximately 150 papers to date, including a number of invited papers, book chapters, and tutorial talks; he is currently coauthoring an undergraduate textbook on digital signal processing. He is currently the Editor-in-Chief of the IET SIGNAL PROCESSING JOURNAL. His research interests include signal-processing algorithms for wireless systems, antenna array techniques, and multihop wireless communications.

Dr. Thompson was a Technical Program Cochair for the IEEE International Conference on Communications, which was held in Glasgow, U.K., in June 2007.

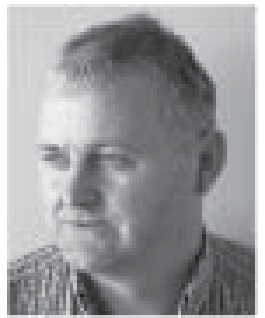

Steve McLaughlin (SM'04) was born in Clydebank, Scotland in 1960. He received the B.Sc. degree in Electronics and Electrical Engineering from the University of Glasgow in 1981 and the Ph.D. degree from the University of Edinburgh in 1989.

From 1981 to 1984 he was a Development Engineer with Barr \& Stroud Ltd. (Glasgow) involved in the design and simulation of integrated thermal imaging and fire control systems. From 1984 to 1986 he worked on the design and development of high frequency data communication systems with MEL Ltd. In 1986 he joined the Dept. of Electronics and Electrical Engineering at the University of Edinburgh as a research fellow where he studied the performance of linear adaptive algorithms in high noise and nonstationary environments. In 1988 he joined the academic staff at Edinburgh, and from 1991 until 2001 he held a Royal Society University Research Fellowship to study nonlinear signal processing techniques. In 2002 he was awarded a personal Chair in Electronic Communication Systems at the University of Edinburgh. His research interests lie in the fields of adaptive signal processing and nonlinear dynamical systems theory and their applications to biomedical and communication systems.

Prof McLaughlin is a Fellow of the Institute of Engineering and Technology, a Senior Member of the IEEE and a Fellow of the Royal Society of Edinburgh. 